\title{
Open KMU: Mit Action Design Research und Design Thinking gemeinsam innovieren
}

\author{
Felix Becker $(\mathbb{D} \cdot$ Michael Meyer $\cdot$ Beke Redlich $\cdot$ Dominik Siemon $(\mathbb{D} \cdot$ \\ Christoph Lattemann
}

Eingegangen: 15. Oktober 2019 / Angenommen: 18. Februar 2020 / Online publiziert: 25. Februar 2020

(C) Der/die Autor(en) 2020

Zusammenfassung Oft reichen die finanziellen Ressourcen und Personalkapazitäten bei KMU nicht für ein strukturiertes Innovationsmanagement aus, das mit denen von großen, multinational agierenden Konzernen mithalten kann. Doch ohne eine beständige und strategische Entwicklung von Innovationen wird die Wettbewerbsfähigkeit der KMU eingeschränkt und gefährdet so nicht nur ihre eigene Position, sondern können auch zur Innovationsbremse in einer Volkswirtschaft werden. Ein möglicher Ansatz ist die Öffnung der Organisation, damit Wissen und Erkenntnisse unterschiedlichster Perspektiven für die Innovationsentwicklung nutzbar werden. Diese Strategie nennt man Open Innovation (OI).

Der folgende Beitrag präsentiert, wie der anwendungsorientierte Forschungsansatz Action-Design-Research (ADR) zielführend Wissenschaftler und Praktiker zusammenbringen kann. Es wird gezeigt, dass durch die Kombination von ADR und dem kreativen Entwicklungsansatz Design Thinking KMU strategisch Innovationen

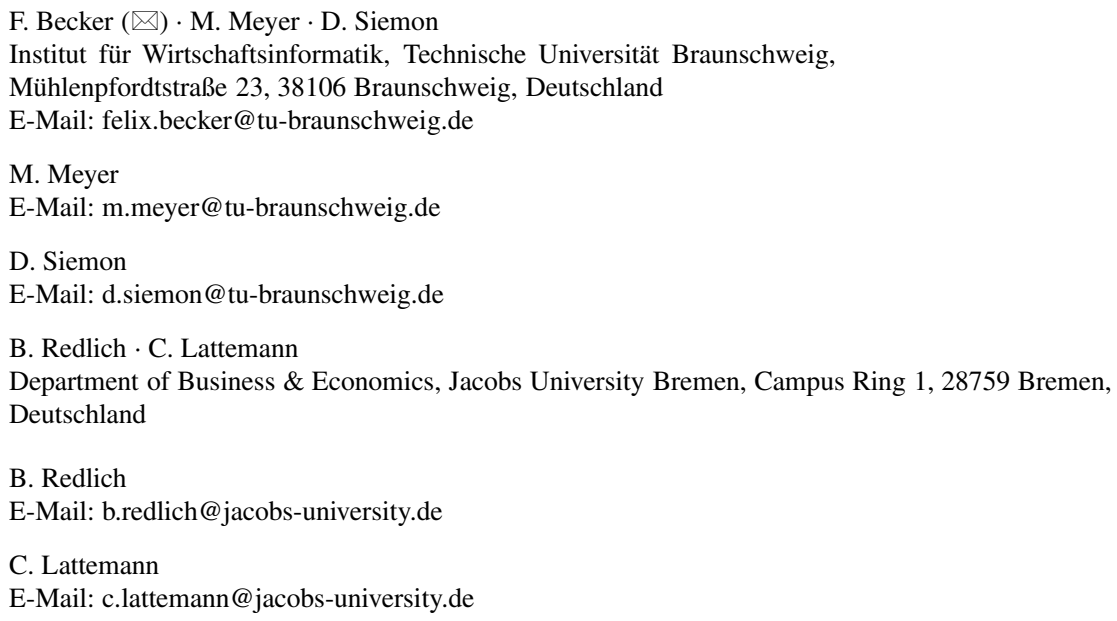


aufbauen und somit ihre Innovationsfähigkeit unter gegebenen Ressourcen steigern können. Dazu werden zunächst die theoretischen Grundlagen von ADR und Design Thinking vorgestellt und anschließend Erfahrungen und Erkenntnisse aus einer 3,5-jährigen Wissenschafts-Praxis-Kollaboration betrachtet. In dem Projekt konnte gezeigt werden, dass die Anwendung von Design Thinking Vorteile birgt, um die Innovationsfähigkeit von KMU zu verbessern.

Schlüsselwörter Action Design Research · Design Thinking · Open Innovation · Wissenschafts-Praxis-Kollaboration

\section{Open SMEs: Collaborative Innovation with Action Design Research and Design Thinking}

Abstract Financial and human resources of small and medium sized enterprises (SMEs) are often insufficient for establishing a structured innovation management that is able to compete with those of large multinational companies. However, without a continuous and strategic development of innovations, the competitiveness of SMEs is restricted and, thus, not only endangers their own position, but can also endanger the economy in terms of innovations. One possible approach is to open up the organization so that knowledge and insights from a wide range of perspectives can be used for innovation development. This strategy is called Open Innovation (OI).

The following article presents an approach on how SMEs can apply the application-oriented research approach Action Design Research (ADR) independently in order to practice OI. It is shown that through the combination of ADR and the creative Design Thinking (DT) approach, SMEs can build a strategic innovation development process and, thus, increase their innovation capacity under given resources. To this end, the theoretical foundations of design-oriented research and design thinking will be presented first, followed by experiences and insights from a 3.5-year science-practice collaboration. In the project it was shown that the application of Design Thinking has advantages to improve the innovative ability of SMEs.

Keywords Action Design Research · Design Thinking · Open Innovation · Science-Practice-Collaboration

\section{Einleitung}

Die zunehmende Globalisierung und digitale Vernetzung versetzten auch einst regionale, kleine und mittlere Unternehmen (KMU) in die Situation, sich mit internationaler Konkurrenz messen zu müssen. Die Digitalisierung birgt zwar für die Unternehmen viele Vorteile, wie die einfache und kostengünstige Erschließung neuer Märkte und Zielgruppen, allerdings auch neue Herausforderungen. Neben neuen Absatzmöglichkeiten entsteht ein immenser Innovationsdruck, gegen den die Unternehmen kaum bestehen können. Neue, agile Forschungs- und Entwicklungsmetho- 
den werden heute bereits erfolgreich von Konzernen eingesetzt (Rauth et al. 2014). Diese bedürfen jedoch eines spezifischen Wissens und finanzieller Ressourcen, über die KMU meistens nicht verfügen. Um weiterhin konkurrenzfähig zu bleiben, bedarf es einer Möglichkeit für KMU ein ressourcenschonendes Innovationsmanagement zusammen mit einem Mindset, das auf Lernen und Kundenbedürfnisse ausgerichtet ist, $\mathrm{zu}$ implementieren.

Eine Möglichkeit, auch ohne eine ressourcenintensive Forschungs- und Entwicklungsabteilung kontinuierlich innovative und kundenorientierte Lösungen zu generieren, ist der Ansatz der Open Innovation (OI). OI wird definiert als ein verteilter Innovations- und Ideengenerierungsprozess, der auf einem zielorientierten Management von Wissensflüssen über Unternehmensgrenzen hinweg basiert. Dabei steht eine Zusammenarbeit mit verschiedenen Partnern, wie externen Forschungseinrichtungen, Universitäten, Wissensgemeinschaften und insbesondere Kunden im Mittelpunkt (Chesbrough 2006). Kerngedanke der OI ist die Öffnung der Organisation, damit die Expertise externer Stakeholder mit eingebunden werden kann, sowie interne und externe Wissensflüsse kombiniert und nutzerzentrierte Innovationen ermöglicht werden.

Oftmals mangelt es KMU an Erfahrung und Wissen im Bereich der OI, um diesen Ansatz zielführend und gewinnbringend einzusetzen. Hier bietet Action Design Research (ADR) (Sein et al. 2011) eine Möglichkeit, anhand eines in der Wissenschafts-Praxis-Kollaborationen erprobten und etablierten Rahmenwerks, innovative und kundenorientierte Lösungen zu entwickeln und gleichzeitig eine adäquate $\mathrm{Zu}$ sammenarbeit innerhalb der Organisation zu verstetigen, die darüber hinaus im besten Fall eine Auswirkung auf die Unternehmenskultur hat. Der vorliegende Beitrag beschreibt eine Möglichkeit, wie mit ADR als Rahmenwerk, verbunden mit Design Thinking (DT) als Ansatz, OI in Unternehmen erfolgreich angewendet werden kann.

Im Folgenden werden die theoretischen Grundlagen aktions- und gestaltungsorientierter Forschung sowie die des DT vorgestellt. Danach wird dann auf die konkrete Anwendung von DT-Workshops innerhalb des ADR-Rahmenwerks eingegangen. Der Beitrag schließt mit der Vorstellung einer Fallstudie, in der das beschriebene Vorgehen angewendet wurde. Hierbei konnte gezeigt werden, dass die Anwendung des ADR-Rahmenwerks in Verbindung mit DT Vorteile birgt, um die Innovationsfähigkeit von KMU zu verbessern.

\section{Action Design Research}

Im Bereich der gestaltungsorientierten Wirtschaftsinformatik (WI) ist die Erarbeitung von Problemlösungen ein etabliertes Forschungsvorgehen. Hierbei entstehen Artefakte, die innovative, auf wissenschaftlichen Theorien aufbauende Lösungen für real existierende Probleme hervorbringen. Das Artefakt kann vielfältige Ausprägungen annehmen. Diese Ausprägungen können beispielsweise prototypisch entwickelte Software oder auch neue Methoden und Modelle sein. Eine strukturierte Vorgehensweise, um solche Artefakte zu entwickeln ist das Forschungsparadigma Design Science Research (DSR). 


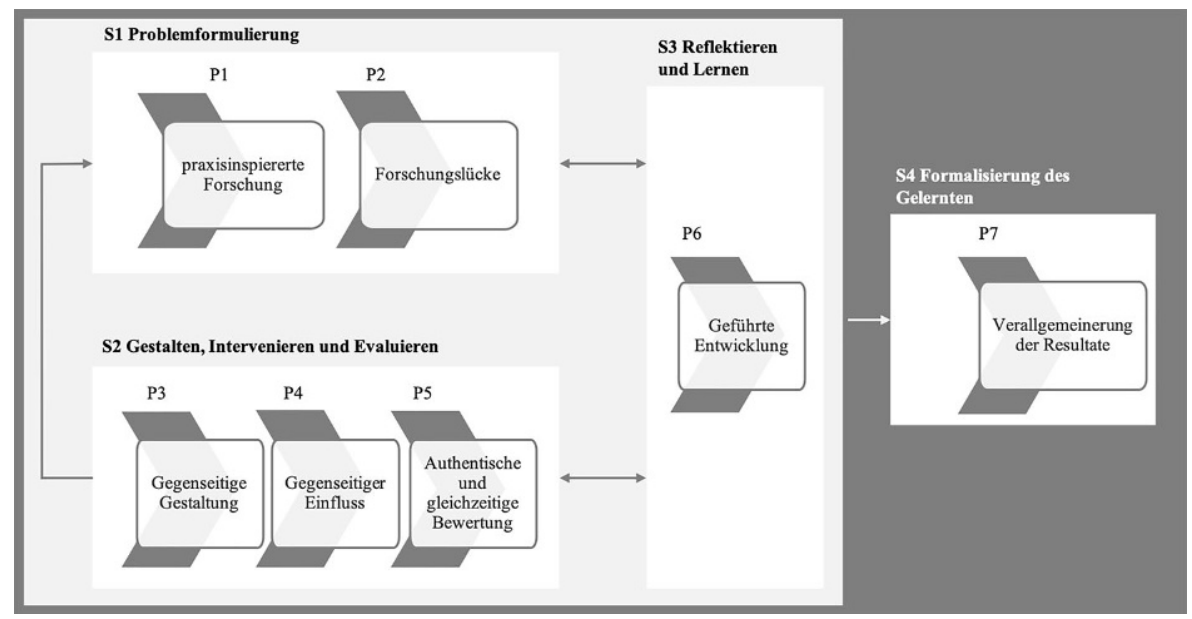

Abb. 1 ADR-Vorgehen nach Sein et al. (2011)

Hevner et al. (2004) schlagen dazu ein Modell vor, das darauf abzielt, eine rigorose Anwendung dieses Forschungsparadigmas zu gewährleisten, indem sie auf die Bedeutung einer angemessenen Relevanzermittlung und systematischen Bewertung hinweisen. Ein Kritikpunkt von DSR ist jedoch, dass dabei der praktische Kontext in dem ein Problem auftritt, nicht ausreichend mit einbezogen wird und somit auch das entwickelte Artefakt bzw. die Lösung den Problemkontext nicht ausreichend berücksichtigt. ADR stellt eine Weiterentwicklung des DSR-Paradigmas dar, die dieser Kritik versucht entgegenzuwirken. ADR ist eine Forschungsmethodik, die eine Kombination aus DSR und der sozialwissenschaftlich geprägten Action Research (AR) darstellt. Während DSR auf der Entwicklung innovativer Artefakte aus der Perspektive eines Problems basiert, setzt ADR bei einer sozialen Problemsituation im unternehmerischen Sinn an (Iivari und Venable 2009).

Die Besonderheit von ADR ist, dass die Integration externen Wissens nicht bei dem Einholen von Informationen bei externen Stakeholdern aufhört, sondern Forscher und Praktiker in einem Team zusammen auf ein gemeinsames Ziel hinarbeiten - sie kollaborieren. Die Kollaboration wird hierbei durch praktische, wiederkehrende Interventionen realisiert.

Durch die Kollaboration von Teams in einem realen Kontext wird das generierte Artefakt zum Ensemble-Artefakt weiterentwickelt. Das Ensemble-Artefakt kann vielfältige Formen wie Konzepte, Dienstleistungen, Prozesse oder Produkte annehmen (Maccani et al. 2015). Das Ensemble-Artefakt entsteht nach und nach durch die wiederkehrenden Interventionen. Dabei nimmt es Bezug auf die Besonderheiten der Problemsituation und erweitert somit die ausschließlich IT-getriebene Artefaktperspektive von DSR (Goldkuhl 2013).

Um die Anwendung des ADR-Vorgehens zu ermöglichen und eine Vergleichbarkeit mit anderen Projekten herzustellen, haben Sein et al. (2011) ein Rahmenwerk entwickelt, das Benutzer durch Stufen (S) und dazugehörigen Prinzipien (P) leitet. Abb. 1 zeigt das ADR-Rahmenwerk von Sein et al. 
Um ADR überhaupt durchführen zu können, muss initial das Engagement von Unternehmen und Forschern sichergestellt werden (Sein et al. 2011). Der Beginn eines ADR-Vorgehens besteht in der Problemformulierung (S1), die sich aus praxisinspirierter Forschung (P1) und einer zugrundeliegenden Forschungslücke (P2) und Forschungsfragen zusammensetzt. Die identifizierten Probleme werden in einem Bündel zusammengeführt, sowie jeweilige Herausforderungen aus der Praxis und forschungsbasierte Implikationen zusammengefasst. Zudem wird die Artefaktentwicklung initialisiert (P2), wobei die Identifizierung relevanter Technologien hier im Vordergrund steht.

Sind die Prinzipien von S1 grundlegend erfüllt, folgt S2, welche aus Gestalten, Intervenieren und Evaluieren (GIE) besteht. Die drei Elemente des GIE werden durchweg als Einheit verstanden, wobei die Einheit GIE über das gesamte ADRVerfahren iterativ wiederholt wird. Die Einheit von GIE muss passend zum Problemkontext eine bestimmte Form annehmen. Durch die Tätigkeiten im GIE wird direkt in die Arbeitsweise der Organisation eingegriffen und die Erkenntnisse können im Nachhinein evaluiert werden. Die Evaluation kann zum Beispiel in Form von Interviews oder Nutzerbefragungen realisiert werden. Durch den grundlegenden Ansatz der aktiven Kollaboration von Stakeholdern sollten das Vorgehen in S2 durch Teilnehmer mit unterschiedlichen Rollen, Expertisen und Kompetenzen ausgeführt werde, sodass sich Teilnehmer gegenseitig positiv beeinflussen können (P4). GIE wird mehrmals und somit in Zyklen durchgeführt, damit je nach gewonnen Erkenntnissen Veränderungen im Problemverständnis und die Art der Intervention angepasst werden können (P3). Wieviele GIE-Zyklen durchgeführt werden, hängt vom Umfang des Projekts und des Projektfortschritts ab (P5).

Eine ebenfalls beständige Aufgabe im ADR-Vorgehen ist das Reflektieren und Lernen (S3). Dabei sind alle Teilnehmer aufgefordert, die Verbesserung der initialen Problemsituation in ihrer Gesamtheit zu betrachten und sich nicht in den kurzfristigen Veränderungen durch beispielsweise den GIE-Zyklus zu verlieren. Hierzu gehört gleichermaßen die Reflektion über die Artefaktgestaltung, wie auch dessen Anpassung, sowie die Reflektion über die Einhaltung aller Prinzipien zur Berücksichtigung des Projektziels (Sein et al. 2011).

Neben der Reflektion, ist im ADR auch die „Formalisierung des Gelernten“ (S4) ein Bestandteil, der sich auf die Verallgemeinerung und Veröffentlichung von Zwischenergebnissen und Erkenntnissen bezieht. Diese Verallgemeinerung und Veröffentlichung ermöglicht es dem Entwicklungsteam, sich Feedback von anderen Stakeholdern einzuholen. Die Formalisierung und Verbreitung des sukzessiv Erlernten kann auch als Grundlage zur „Verallgemeinerung der Resultate“ (P7) genutzt werden, die üblicherweise erst zum Ende des ADR-Vorgehens entwickelt werden können. Die Resultate können dann in Form von Konzepten übertragen werden, die auch für vergleichbare Kontexte einen Mehrwert bieten, indem die Gestaltungsprinzipien herausgearbeitet werden. S4 beendet das ADR-Vorgehen, welches der verbesserten Situation als Antwort auf die Problemstellung in Form eines Ensemble-Artefakts darstellt.

S2, die die eigentlichen Tätigkeiten in der Artefaktentwicklung beinhaltet, bleibt auch im ausführlich beschrieben Rahmenwerk von Sein et al. (2011) eher abstrakt. Es wird nicht klar, welche Möglichkeiten es zur praktischen Umsetzung der Stufe 
Gestalten, Intervenieren, Evaluieren gibt. Für die Durchführung der Stufe schlagen wir deshalb den Einsatz von DT-Workshops vor, was auch dem Ansatz von Veling et al. (2016) folgt. Das folgende Kapitel beschreibt DT zuerst allgemein, um dann detaillierter darauf einzugehen, wieso sich dieses Vorgehen für die Anwendung in mit ADR durchgeführten OI Projekten eignet.

\section{Design Thinking}

Design Thinking (DT) ist ein nutzerorientierter und partizipatorischer Innovationsansatz, der in Workshop-Formaten durchgeführt wird (Brown 2008). DT-Workshops eignen sich, um innovative Lösungen für komplexe Probleme zu entwickeln (Johansson-Sköldberg et al. 2013). Eine Besonderheit des DT-Ansatzes ist es, dass die Kollaboration lösungsoffen begonnen wird, damit noch keine Verbesserungsideen bestehen und gemeinsam ein Problemkontext definiert werden kann. Gegenüber anderen Ansätzen zur Lösung komplexer Probleme zeichnet sich DT vor allem durch die umfassende Integration aller Stakeholder aus und passt somit exakt in das ADRVorgehen. Wichtig für die erfolgreiche Durchführung eines DT-Workshops ist dabei eine möglichst heterogene Gruppe von Teilnehmern. Dies sollte wiederum durch die Teilnahme externer Stakeholder sichergestellt werden. Durch den hohen Integrationsgrad, der Nutzerzentrierung, dem Fokus auf die Lösung komplexer Probleme, dem Ziel, innovative Lösungen zu schaffen, sowie die Wirksamkeit der Lösung zu bewerten, eignen sich DT-Workshops gut für das Kollaborieren mit dem gemeinsamen Ziel der Innovationsentwicklung.

Der DT-Ansatz besteht grundsätzlich aus drei Elementen: dem DT-Prozess, der aus verschiedenen iterativ durchlaufenden Phasen besteht; den DT-Methoden, die jeweils passend zu den Phasen ausgewählt und eingesetzt werden; und dem DTMindset, welches die Herangehensweise der Zusammenarbeit beschreibt (Brenner et al. 2016).

Es existieren unterschiedliche Versionen des DT-Ansatzes. Für das in diesem Beitrag beschriebene Vorgehen lässt sich ein Ansatz, der speziell für KMU in den industrienahen Dienstleistungen entwickelt wurde, besonders gut anwenden: das sogenannte DETHIS-Verfahren. Dieser Prozess enthält die Phasen Design Challenge, Verstehen, Beobachten, Standpunkt definieren, Ideen entwickeln, Prototyp, Test und Implementierung, die speziell auf die Belange von KMU eingehen. Für die Initialisierung, sowie die Anschlussfähigkeit der Lösungen sind eigene Phasen vorgesehen. Dies ist bei fehlenden Forschungs- und Entwicklungsabteilungen vorteilhaft, um KMU zu befähigen, systematisch zu innovieren (Fischer et al. 2019).

Die Basis eines DT-Workshops ist die sogenannte Design-Challenge, welche als eine Art Aufgabenstellung fungiert. Die Design-Challenge wird dabei als eine „How Might We" beziehungsweise eine ,Wie können wir es schaffen"-Frage formuliert und initialisiert einen DT-Workshop (Siemon et al. 2018).

In den Prozessschritten Verstehen und Beobachten ist es erforderlich, ein besseres Verständnis für das Problem und den oder die dahinterstehenden Menschen aufzubauen. Mögliche Methoden, um entsprechende Informationen zu erhalten sind allgemeine Recherchen, Interviews oder Beobachtungen. Ziel dieser Phasen ist die 
Identifizierung von Stakeholdern und der Aufbau von Empathie zum potentiellen Nutzer beziehungsweise der Nutzergruppe (Köppen und Meinel 2015).

Der Schritt Standpunkt definieren hat das Ziel, eine möglichst exakte Definition des Problems beziehungsweise des zu bearbeitenden Problemraums aus der Nutzerperspektive zu schaffen. Alle folgenden Schritte des DT-Workshops bauen darauf auf, dass alle Teilnehmer einer neu entwickelten, nutzerzentrierten Problemstellung folgen, die gemeinschaftlich erarbeitet wurde.

Die Phase Ideen entwickeln zielt darauf ab, eine möglichst hohe Anzahl vielfältiger Ideen zu generieren. Typische Methoden in diesem Schritt sind BrainstormingTechniken in verschiedenen Formen. Die Teilnehmer des DT-Workshops werden dabei ermutigt, auf Einschränkungen wie Kosten oder Umsetzbarkeit zu achten.

Die vielversprechendsten Ideen werden in den Prozessschritt Prototyp übertragen. In diesem Schritt versuchen die Teilnehmer, mit verschiedensten Materialien die Ideen in Form einfacher Prototypen umzusetzen. Das Ziel dieser Phase ist es, eine sichtbare und/oder greifbare Version einer Idee umzusetzen (Potthoff et al. 2018). Die Relevanz der erstellten Prototypen wird schließlich in dem Prozessschritt Test erprobt. Dabei können verschiedene Testszenarien, wie beispielsweise einfaches Ausprobieren bis hin zu komplexen Use Cases oder Rollenspiele zum Einsatz kommen. Hierbei kommt es darauf an, das Feedback der Nutzer einzuholen, damit die Innovation einen tatsächlichen Wert für die potenziellen Nutzer aufweist. Ist ein Test positiv ausgefallen, kann in der Phase Implementierung das weitere Vorgehen eines Unternehmens geplant werden, um die Innovation bis zur Marktreife zu entwickeln. Neben dem DT-Prozess und den DT-Methoden ist das DT-Mindset die Richtlinie, wie im Team zusammengearbeitet wird (Brenner et al. 2016). Bestandteil des Mindset ist beispielsweise eine Toleranz gegenüber Mehrdeutigkeit, sodass der Mehrwert unterschiedlicher Perspektiven einfließen kann (Carlgren et al. 2016). Ebenfalls ist das gemeinsame Visualisieren, flache Hierarchien und eine empathische Einstellung, um die Nutzerzentrierung zuzulassen, Bestandteil des DT-Mindset. Das kreative Zusammenarbeiten in diversen Teams innerhalb des DT-Ansatzes ist oftmals konträr zu gängigen Arbeitsroutinen in Unternehmen, was die Bedeutung des DT-Mindset für den Gesamtprozess noch stärker in den Fokus rückt.

\section{Action Design Research und Design Thinking}

Schon bevor der Begriff ADR in der Forschung etabliert wurde, beschäftigte sich ein schwedisches Forscherteam mit dem Einsatz von Kreativmethoden in einem Forschungsprojekt in der Automobilindustrie. Dieses Vorhaben ist somit quasi der Vorgänger des ADR-Ansatzes im Bereich des Innovationsmanagements (Almefelt et al. 2003). Hierbei konnten die Wissenschaftler feststellen, dass der Einsatz von Kreativmethoden in kollaborativen Entwicklungsprozessen deutliche Prozessvorteile birgt. Darüber hinaus stellten Petersson und Lundberg (2016) fest, dass sich der Einsatz von ADR besonders für Projekte eignet, bei denen die Innovationsentwicklung einen hohen Stellenwert einnimmt. Sie konnten zeigen, dass der Einsatz von Kreativmethoden als Form der Intervention im ADR-Framework die Kooperation 
der Projektteilnehmer untereinander und den Einsatz für das vorher festgelegte, gemeinsame Ziel erhöht.

Während ADR eine Forschungsmethodik ist, die mit Hilfe eines dezidierten Vorgehens in Form eines Rahmenwerks die Ausführung, sozusagen das Projektmanagement, gestaltet, ist DT ausschließlich eine Innovationsansatz und keine Forschungsmethodik. Durch die Vorgabe im ADR-Vorgehen, dass die Zusammenarbeit durch Interventionen, gestaltet wird, kann DT in das ADR-Vorgehen integriert werden (Veling et al. 2016).

Für eine Vorgehensempfehlung in Stufe 2 des ADR-Ansatzes eignen sich DTWorkshops, da große Ähnlichkeiten zwischen dem DT-Vorgehen und Gestalten, Intervenieren und Evaluieren bestehen; beispielsweise die Durchführung von interaktiven, multidisziplinären und kollaborativen Workshops mit einem Schwerpunkt auf eine strikte Nutzerorientierung. Eine Intervention kann aber auch ein UX/UI-Test, eine Beobachtung oder die Erprobung eines neuen Prozesses im Unternehmen sein. Die Interventionen haben das Ziel, Wissen zu generieren, was durch unterschiedliche Perspektiven, dem institutionellen Kontext und einen Austausch von internen und externen Wissensflüssen ermöglicht werden soll. Das Workshop-Format bietet dabei den Vorteil, dass ein Projektteam bei Bedarf aus unterschiedlichen Abteilungen des Unternehmens gebildet werden kann. Somit kann auf eine abgegrenzte Innovationsabteilung verzichtet werden, was letztendlich wichtige Ressourcen spart und eine unternehmensweite Zusammenarbeit sicherstellt.

Die Nutzung von DT als Interventionen im ADR wird auch durch Veling et al. (2015, 2016) unterstützt. Die Verwendung von DT-Workshops kann somit als eine gute Form der Intervention in ADR angesehen werden (Veling et al. 2015, 2016; Becker et al. 2019). Die hier skizzierten Arbeiten von Almefelt et al. (2003) und Peterson und Lundberg (2016) unterstreichen, dass die Anwendung von ADR für Innovationsansätze geeignet ist, bei denen die Nutzeranforderungen in kreativen Umgebungen für die Zusammenarbeit im Mittelpunkt stehen.

\section{Open Innovation mit ADR und DT in einer Wissenschafts-Praxis- Kollaboration}

Die vorgeschlagene Integration von ADR und DT innerhalb eines OI-Projekts konnte bereits erfolgreich in einem 3,5jährigen Konsortialprojekt erprobt werden (Redlich et al. 2019). Im Folgenden sollen die Rahmenbedingungen des Projekts zunächst erläutert werden, um anschließend die gewonnenen Erkenntnisse darzustellen. Das Hauptziel des Projekts war es Möglichkeiten zu finden, wie die Innovationskraft von KMU nachhaltig gesteigert werden kann. Mit Hilfe von DT-Workshops sollten 1. unter Anwendung eines digitalisierten DT-Vorgehens innovative Dienstleistungsansätze für die beteiligten Praxispartner entwickelt werden und 2. ein innovationsund kundenorientiertes Mindset in den Unternehmen nachhaltig verankert werden (ebd.).

Neben den vier universitären Partnern, die die Möglichkeiten der Digitalisierung von DT beziehungsweise die aktuellen Herausforderungen im Innovationsmanagement von industrienahen Dienstleistungen beforschten, waren auch drei Unterneh- 
men in zwei verschiedenen Rollen im Projekt beteiligt. Zwei der Unternehmen dienten als Anwendungspartner (U1 und U2), wobei das dritte Unternehmen als Softwareentwickler einer digitalen DT-Plattform fungierte (U3). Das Vorgehen innerhalb des Projektes orientierte sich dabei an dem ADR-Rahmenwerk von Sein et al. (2011) (siehe Abb. 1). Zu Beginn des Projektes wurden die aktuellen Prozesse des Innovationsmanagements von U1 und U2 untersucht, mit dem Ergebnis, dass beide Unternehmen kein oder nur bedingt ein systematisches Innovationsmanagement betreiben und dabei keine Kunden in den Prozess mit einbeziehen; also keine OI verfolgen, welches ein typisches Vorgehen von KMU darstellt. Dies führte unter anderem dazu, dass keine bzw. nur wenige Innovationen kundennah entwickelt und realisiert wurden. Mit Beginn des Projektes wurden daraufhin die in Stufe 2 vorgesehenen Tätigkeiten mit Hilfe einer Reihe von DT-Workshops durchgeführt. Dabei wurden Lösungen und konkrete Innovationen mit Kunden in den Unternehmen anhand des DETHIS-Verfahrens entwickelt. Durch Experteninterviews und Beobachtungen während der Workshops konnte vor allem herausgefunden werden, dass das Vorgehen als neuartig, innovationsfördernd, motivierend und kollaborativ beschrieben wurde. Es stellte nicht nur einen völlig neuen und zuvor unbekannten Ansatz für die Problemlösung und Innovation im Unternehmen und der Organisationsentwicklung dar, sondern bot mit seinem spielerischen Hands-On-Charakter auch eine willkommene Abwechslung. Dabei konnten Methoden erlernt und angewendet werden, sowie der Einsatz von digitalen und analogen Tools erprobt werden. Die Workshops wurden größtenteils in extra dafür eingerichteten Räumen, so genannten DT-Workspaces durchgeführt. Diese zeichnen sich durch modulare und mobile Möbel, sowie viel Raum für Zeichnungen, Notizen und anderen Visualisierungen in Form von Whiteboards und Pinnwänden aus. Für die Prototyping-Phase des DT wurde darüber hinaus ausgiebig Material (Lego, Bastelmaterial, Mockups etc.) zur Verfügung gestellt, wodurch schnell und einfach erste Ideen entwickelt werden konnten.

Bei beiden Unternehmen führten die wiederkehrenden Interventionen zu einer nachhaltigen Organisationsveränderung. Das Mindset der Unternehmen für einen offenen und kollaborativen Innovationsprozess nach dem DETHIS-Verfahren konnte dadurch geschaffen werden. Vor allem U1 profitierte von der Auseinandersetzung mit neuen Innovationsmethoden und schuf sogar eine neue Stelle; den Innovationsmanager. Diese Position ist zuständig für die Fortführung der Innovationsarbeit im Unternehmen. Auch U2 berichtet von positiven Veränderungen. Die Einbeziehung externer Unternehmensstakeholder, wie Kunden und Zulieferer, führte auch hier zu innovativen Entwicklungen und zur Veränderung von Prozessen und Produkten.

\section{Zusammenfassung und Ausblick}

In diesem Artikel wurde aufgezeigt, wie sich Wissenschafts-Praxis-Kollaboration nutzen lassen, um die Innovationsfähigkeit von KMU langfristig zu steigern. Hierzu wurden zunächst die theoretischen Grundlagen von ADR und DT beschrieben und dann aufgezeigt, wie sich DT in der Stufe 2, (,Gestalten, Intervenieren, Evaluieren“) des ADR-Vorgehens nach Sein et al. (2011) nutzen lässt. DT eignet sich dabei 
besonders wegen der prozessual sowieso vorgesehenen Integration externer Stakeholder in den Innovationsprozess, durch den iterativen Charakter der Workshops und dem Fokus auf die Lösung komplexer Fragestellungen. Unternehmen können so dem Rahmenwerk von ADR folgen, mit DT-Workshops ausgestalten und dadurch OI praktizieren. Darüber hinaus ist DT gut dokumentiert, was die Anwendung innerhalb von OI-Bemühungen in KMU vereinfacht. Online lassen sich detaillierte Beschreibungen des DT-Prozesses, sowie einzelner Methoden finden ${ }^{1}$. Die Verinnerlichung des bereits angesprochenen Mindset nimmt mehr Zeit in Anspruch, was den Vorteil des iterativen Vorgehens bestärkt.

Die grundsätzlichen Vorteile, die das hier vorgestellte Vorgehen für die Forschung bietet sind erstens die Möglichkeit, Wissen aus dem universitären Kontext in Unternehmen mit einem direkten Praxisbezug zu transferieren und zweitens, die gewonnen Erkenntnisse wieder aus der geschlossenen Wissensbasis der Unternehmen zurück in die wissenschaftliche Wissensbasis zu überführen, da das in Unternehmen generierte Wissen dem wissenschaftlichen Diskurs aus Unternehmensinteressen oft vorenthalten wird. Somit können alle Akteure von dem vorgeschlagenen Vorgehen profitieren.

Dass der hier vorgeschlagene Ansatz in einer Wissenschafts-Praxis-Kollaboration gut funktioniert, konnte in der beschriebenen Fallstudie gezeigt werden. Dabei war es möglich, die Innovationsfähigkeit der Unternehmen zu stärken. Der vorliegende Artikel leistet somit einen Beitrag zur praktischen Anwendung von ADR innerhalb von Wissenschafts-Praxis-Kollaboration und zeigt auf, dass sich durch diese Art der Durchführung von ADR praxisrelevante Forschung und neue Erkenntnisse einerseits und strategische Vorteile für KMU andererseits gleichzeitig realisieren lassen.

Funding Open Access funding provided by Projekt DEAL.

Open Access Dieser Artikel wird unter der Creative Commons Namensnennung 4.0 International Lizenz veröffentlicht, welche die Nutzung, Vervielfältigung, Bearbeitung, Verbreitung und Wiedergabe in jeglichem Medium und Format erlaubt, sofern Sie den/die ursprünglichen Autor(en) und die Quelle ordnungsgemäß nennen, einen Link zur Creative Commons Lizenz beifügen und angeben, ob Änderungen vorgenommen wurden.

Die in diesem Artikel enthaltenen Bilder und sonstiges Drittmaterial unterliegen ebenfalls der genannten Creative Commons Lizenz, sofern sich aus der Abbildungslegende nichts anderes ergibt. Sofern das betreffende Material nicht unter der genannten Creative Commons Lizenz steht und die betreffende Handlung nicht nach gesetzlichen Vorschriften erlaubt ist, ist für die oben aufgeführten Weiterverwendungen des Materials die Einwilligung des jeweiligen Rechteinhabers einzuholen.

Weitere Details zur Lizenz entnehmen Sie bitte der Lizenzinformation auf http://creativecommons.org/ licenses/by/4.0/deed.de.

\footnotetext{
1 https://dschool.stanford.edu/resources.

http://www.designkit.org/methods.

https://designthinking-methoden.de.
} 


\section{Literatur}

Almefelt L, Sutinen K, Malmqvist J (2003) Computer support for systematic design applied in a crossfunctional commercial concept development project. Concurr Eng 11:107-120. https://doi.org/10. 1177/1063293X03035425

Becker F, Meyer M, Siemon D, Robra-Bissantz S (2019) Taking action: extending participatory action design research with design thinking. In: Humanizing technology for a sustainable society. University of Maribor Press, Maribor, Slowenien, S 1073-1084

Brenner W, Uebernickel F, Abrell T (2016) Design thinking as mindset, process, and toolbox. In: Brenner W, Uebernickel F (Hrsg) Design thinking for innovation. Springer, Cham, S 3-21

Brown T (2008) Design Thinking. Harv Bus Rev 86:84-92, 141

Carlgren L, Rauth I, Elmquist M (2016) Framing design thinking: the concept in idea and enactment. Creat Innov Manag 25:38-57. https://doi.org/10.1111/caim.12153

Chesbrough HW (2006) Open innovation: the new imperative for creating and profiting from technology. Harvard Business Press, Boston

Fischer S, Redlich B, Gernreich C, Poeppelbuss J, Lattemann C (2019) Implementation of Design Thinking in an SME. In: ISPIM Conference Proceedings. The International Society for Professional Innovation Management, Manchester, UK

Goldkuhl G (2013) From ensemble view to ensemble artefact-An inquiry on conceptualisations of the IT artefact. Syst Signs Actions 7:49-72

Hevner, March, Park, Ram (2004) Design science in information systems research. Mis Q 28:75. https:// doi.org/10.2307/25148625

Iivari J, Venable J (2009) Action research and design science research - Seemingly similar but decisively dissimilar. In: Proceedings of the 17th European Conference on Information Systems, ECIS 2009, Verona, Italy. S 1642-1653

Johansson-Sköldberg U, Woodilla J, Çetinkaya M (2013) Design thinking: past, present and possible futures. Creat Innov Manag 22:121-146. https://doi.org/10.1111/caim.12023

Köppen E, Meinel C (2015) Empathy via design thinking: creation of sense and knowledge. In: Plattner H, Meinel C, Leifer L (Hrsg) Design thinking research. Springer, Cham, S 15-28

Maccani G, Donnellan B, Helfert M (2015) Action design research: a comparison with canonical action research and design science. DESRIST 2015.

Petersson AM, Lundberg J (2016) Applying action design research (ADR) to develop concept generation and selection methods. Procedia Cirp 50:222-227. https://doi.org/10.1016/j.procir.2016.05.024

Potthoff T, Siemon D, Wilms K et al (2018) Collaborative service blueprinting for design thinking: evaluation of a digital prototype

Rauth I, Carlgren L, Elmquist M (2014) Making it happen: legitimizing design thinking in large organizations. Des Manag J 9:47-60. https://doi.org/10.1111/dmj.12015

Redlich B, Becker F, Fischer S et al (2019) Das DETHIS-Verfahren: Design Thinking für das ServiceEngineering in kleinen und mittleren Unternehmen. In: Stich V, Schumann JH, Beverungen D et al (Hrsg) Digitale Dienstleistungsinnovationen. Springer, Berlin, Heidelberg, S 73-88

Sein MK, Henfridsson O, Purao S et al (2011) Action design research. Manag Inf Syst Q 35:37. https:// doi.org/10.2307/23043488

Siemon D, Becker F, Robra-Bissantz S (2018) How might we? From design challenges to business innovation. J Creat Bus Innov 4:96-110

Veling L, Thornley C, Murnane S et al (2015) Selecting a suitable methodology for designing innovative solutions to support capability improvement in a complex organisational context as part of an industry-academic collaboration Bd. 21

Veling L, Quillan LM, Browne A et al (2016) Use it or lose it: embodying practice in action design research (ADR). ICIS 2016 Proceedings, S 1-16 